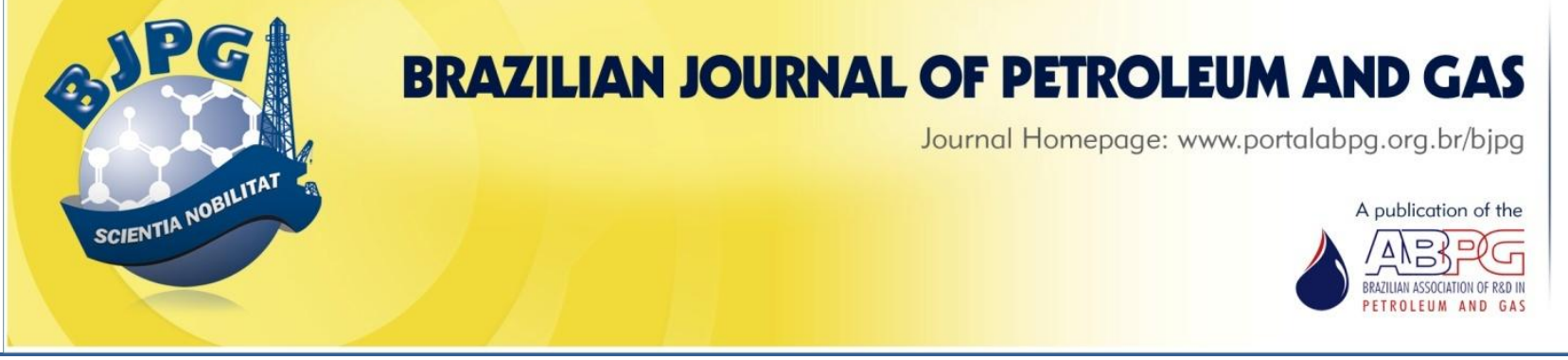

\title{
IMPROVING HYDROCYCLONE GEOMETRY FOR OIL/WATER SEPARATION
}

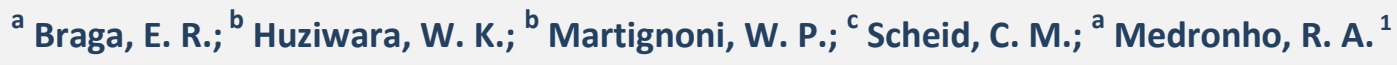 \\ ${ }^{a}$ Federal University of Rio de Janeiro, Dept. of Chem. Eng., School of Chemistry, Brazil \\ ${ }^{b}$ Cenpes/Petrobras, Research Center Leopoldo Miguez de Mello, Rio de Janeiro, Brazil \\ ${ }^{c}$ Federal Rural University of Rio de Janeiro, Dept. of Chem. Eng., Institute of technology, Brazil
}

\begin{abstract}
Hydrocyclones use centrifugal fields to promote the separation of a disperse phase from a continuous phase. Hydrocyclones have advantages in comparison with others separation devices due to their low cost of manufacturing, installation, maintenance, and simple operation. The purpose of this work was to develop a hydrocyclone for water treatment of refinery streams with an oil mass fraction of $1 \%$. CFD simulations of hydrocyclones were carried out using a rotatable central composite design with response surface methodology to determine the values of overflow and underflow diameters that would produce a high separation efficiency and low split ratio. The results show that the best values are $10 \mathrm{~mm}$ for overflow and $15 \mathrm{~mm}$ for underflow, resulting in a split ratio of 13.1 and an oil separation efficiency of $72 \%$ for a $25 \mu \mathrm{m}$ oil drop size.
\end{abstract}

\section{KEYWORDS}

hydrocyclone; CFD; experimental design; oil water separation

\footnotetext{
${ }^{1}$ To whom all correspondence should be addressed. Address: Escola de Química/UFRJ, Av. Athos da Silveira Ramos 149, Centro de Tecnologia, Bloco E, sala E-207, Ilha do Fundão, Rio de Janeiro - RJ, Brazil. ZIP Code: 21941-909 | Phone: +55(21)3938-7635 |e-mail: $\underline{\text { medronho@eq.ufri.br }}$ doi:10.5419/bjpg2015-0012
} 


\section{INTRODUCTION}

The increasing water pollution is a global concern, considering that water is an increasingly scarce and vital resource. Therefore, it is important to improve technologies for the treatment of effluents by disposing off and recycling water.

The hydrocyclone presents advantages in comparison to others separation equipments. Among them, it may be pointed out the versatility in applications, no moving parts, reduced size in comparison to other separators, high capacity, simple operation, and low costs of manufacturing, installation, and maintenance (Svarovsky, 2000). Operating costs are also low since hydrocyclones work at relatively low pressure drops.

Bai (2009) reported an experimental study of the flow in a hydrocyclone to separate water from oil. He applied the velocimetry technique using two laser Doppler components to measure axial velocity, tangential velocity and velocity fluctuations in a hydrocyclone with $35 \mathrm{~mm}$ diameter. He observed that velocity fluctuations were larger near both the center and the wall. This study was useful to understand the separation mechanism of oil removal in a hydrocyclone.

The purpose of this work is to develop a hydrocyclone for separating oil present in oily waters of typical refinery streams using computational fluid dynamics (CFD).

To analyze the performance of the hydrocyclone, three variables were obtained, the water flow ratio $R_{w}$ (Eq. 1), the grade efficiency $G$ (Eq. 2), and the reduced grade efficiency $G^{\prime}$ (Eq. 3).

$R_{w}=\frac{W_{w o}}{W_{w i}}$

$G=\frac{W_{o o}}{W_{o i}}$

$G^{\prime}=\frac{G-R_{w}}{1-R_{w}}$

Where $W_{w o}$ is the water mass flowrate at the overflow, $W_{w i}$ is the water mass flowrate at the feed stream, $W_{o o}$ is the oil mass flowrate at the overflow, and $W_{o i}$ is the oil mass flow at the feed stream.
Delgadillo and Rajamani (2007) proposed some modifications to the classical hydrocyclone geometric proportions aiming at increasing separation efficiency. Further, the authors used CFD to explore alternative geometries. They used the Large Eddy Simulation (LES) as turbulence model and the Lagrangian method for particle tracking. They explored six different geometries and compared them to a standard geometry. At last, they verified that the two of the geometries used had a superior performance than the standard geometry, being candidates for experimental validation.

For the turbulent flow in hydrocyclones, it is important to have an accurate description of the turbulent behavior (Griffiths \& Boysan, 1996). There are several turbulence models that can be used on CFD simulations, such as the $\kappa-\varepsilon$, Reynolds Stress Models, and Large Eddy Simulation. Many researchers have investigated turbulence models (Sommerfeld \& Ho, 2003; Huang et al., 2004; Elsayed \& Lacor, 2011; Bernardo et al., 2006; Gupta \& Kumar, 2007; and Kaya \& Karagoz, 2008).

The $\kappa-\varepsilon$ models, in general, do not describe strong swirling flows properly (Chuah et al., 2006); Wan et al., 2008; and Elsayed \& Lacor, 2011). The Reynolds Stress Models (RSM) are capable of predicting the combined vortex in accordance with experimental data (Elsayed \& Lacor, 2011 and Kaya \& Karagoz, 2008). The Reynolds Stress Models require solving transport equations for each of the Reynolds stress components. The Gibson and Launder Reynolds Stress Model (Gibson \& Launder, 1978) consider pressure strain as linear. The present study used this model.

In a previous study, Freitas (2009) used a reduced factorial experimental design to analyze the influence of geometric parameters of a deoiling hydrocyclone in the split ratio and reduced particle size separation efficiency. The parameters analyzed were diameter of the cylinder section, overflow diameter, underflow diameter, inlet height, vortex finder length, and the length of the conical region (Freitas et al., 2009). They found that the variables with statistical significance regarding separation efficiency were overflow and underflow diameters. These two were the factors evaluated on the experimental design for this work. 


\section{MATERIALS AND METHODS}

The hydrocyclone geometry used in this work is shown in Figure 1 and geometry data are reported in Table 1.

An experimental design (rotatable central composite design with response surface methodology) was used to evaluate the influence of the overflow and underflow diameters (Do and $\mathrm{Du}$, respectively) on the hydrocyclone performance resulting in nine CFD simulations. Table 2 shows the values of Do and Du used in the simulations. The responses were grade efficiency $(G)$ and water flow ratio $\left(R_{w}\right)$.

The experimental design must have between 3 and 5 central points (Calado \& Montgomery, 2003). But simulated values with the same parameters always give the same results, so table 2

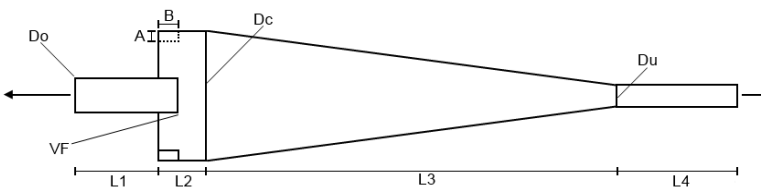

Figure 1. Scheme of a hydrocyclone used in this work.

shows only one value for the central points.

The geometries of the simulated hydrocyclones were built using the software Design Modeler from Ansys. To generate the hexahedral mesh, the software Icem CFD, also from Ansys, was employed. An independent mesh test was carried out and a mesh with approximately 800,000 nodes was found to be ideal to simulate these hydrocyclones (Figure 2).

Table 1. Hydrocyclone geometry data.

\begin{tabular}{lc}
\hline Geometric Variable & Dimensions (mm) \\
\hline Length of inlet (A) & 5 \\
Height of inlet (B) & 23 \\
Length of vortex finder (VF) & 48 \\
Length of overflow tube (L1) & 50 \\
Length of cylindrical session (L2) & 72 \\
Length of conical session (L3) & 810 \\
Length of underflow tube (L4) & 64 \\
Length of the inlet tubes & 40 \\
Total length (L1+L2+L3+L4) & 996
\end{tabular}

Table 2. Variables used in the simulations.

\begin{tabular}{ccccc}
\hline Simulation & $\mathrm{D}_{\mathrm{o}}(\mathrm{mm})$ & $\mathrm{D}_{\mathrm{u}}(\mathrm{mm})$ & $\mathrm{D}_{\mathrm{o}}^{\prime}$ (standardized) & $\mathrm{D}_{\mathrm{u}}{ }^{\prime}$ (standardized) \\
\hline 1 & 5 & 10 & -1 & -1 \\
2 & 5 & 20 & -1 & 1 \\
3 & 15 & 10 & 1 & -1 \\
4 & 15 & 20 & 1 & 1 \\
5 & 2.93 & 15 & -1.414 & 0 \\
6 & 17.07 & 15 & 1.414 & 0 \\
7 & 10 & 7.93 & 0 & -1.414 \\
8 & 10 & 22.07 & 0 & 1.414 \\
9 & 10 & 15 & 0 & 0 \\
\hline
\end{tabular}



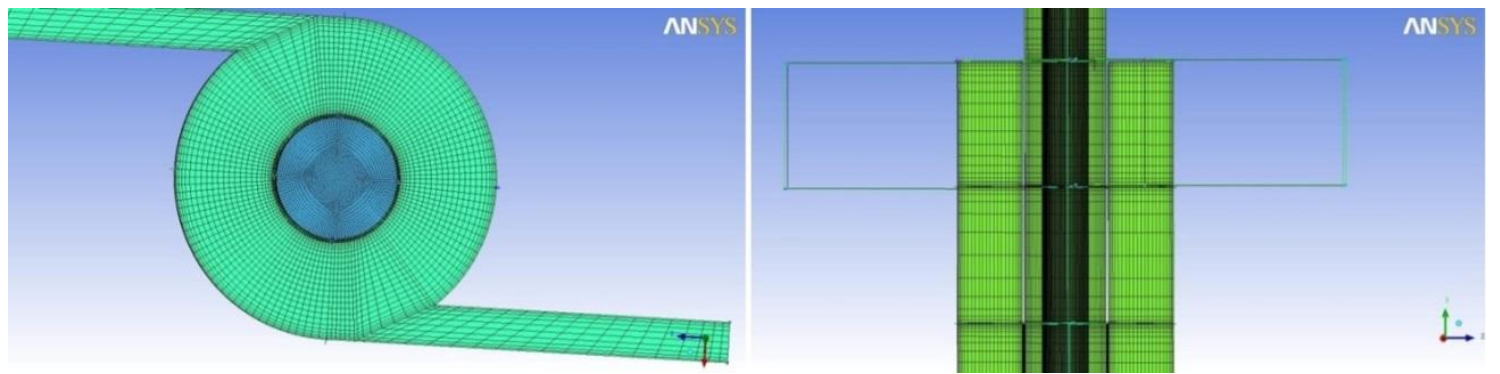

Figure 2. Superior and internal view of the hydrocyclone mesh.

Table 3 presents important conditions used in the simulations.

Initially, simulations used an oil drop size of 100 $\mu \mathrm{m}$, but almost all geometries presented high efficiency making it difficult to compare them. Therefore, the oil drop size was reduced to $25 \mu \mathrm{m}$, which was used in all simulations.

The total time of the simulations was based on the residence time of the fluids in the equipment, which was $0.35 \mathrm{~s}$. It was expected that pressure, efficiency, and water flow ratio would become constant when simulation time reached the triple of the residence time $(1.05 \mathrm{~s})$. In this study it happened close to this time, and the total simulated time was set to $1.13 \mathrm{~s}$. The time step used was $10^{-4}$, which has led to an average Courant number of approximately 2 and a maximum Courant number of 10 . The Courant number is defined as equation 3 . These are acceptable values to ensure stability conditions (Dirgo \& Leith, 1985).

$$
C o=\frac{u \cdot \Delta t}{\Delta x}
$$

Where Co is the Courant number, $\mathrm{u}$ is the fluid speed, $\Delta t$ is the timestep, and $\Delta x$ is the mesh size.

Table 3. Boundary conditions, physical properties of the fluids, and other conditions used in the simulations.

\begin{tabular}{ll}
\hline \multicolumn{1}{c}{ Parameter } & \multicolumn{1}{c}{ Value/Type } \\
\hline Feed volumetric flow rate & $1806 \mathrm{~cm}^{3} / \mathrm{s}$ \\
Water mass fraction & 0.99 \\
Water density & $997 \mathrm{~kg} / \mathrm{m}^{3}$ \\
Oil drop size & $25 \mu \mathrm{m}$ \\
Oil density & $840 \mathrm{~kg} / \mathrm{m}^{3}$ \\
Oil viscosity & $13 \mathrm{cP}$ \\
Overflow absolute pressure & 1 atm \\
Underflow absolute pressure & 1 atm \\
Pressure and velocity coupling & Phase Coupled SIMPLE \\
Turbulence model & Gibson and Launder (1978) \\
Multiphase model & Euler-Euler \\
Momentum interpolation scheme & Second order upwind \\
Volumetric fraction interpolation scheme & First order upwind \\
Turbulent kinetic energy interpolation scheme & Second order upwind \\
Turbulent dissipation rate interpolation scheme & Second order upwind \\
Reynolds stress interpolation scheme & First order upwind \\
Relaxation factor for moment, tensors and continuity & $0.7,0.5$ and 0.8 \\
Time Step & $10^{-4}$ \\
Total simulated time & 1.13 s \\
\hline
\end{tabular}


Table 4. Results of the rotatable central composite design.

\begin{tabular}{ccccc}
\hline Simulation & $\begin{array}{c}\mathrm{D}_{\mathrm{o}}{ }^{\prime} \\
\text { (standardized) }\end{array}$ & $\begin{array}{c}\mathrm{D}_{\mathrm{u}}{ }^{\prime} \\
\text { (standardized) }\end{array}$ & $\begin{array}{c}\mathrm{R}_{\mathrm{w}} \\
(\%)\end{array}$ & $\begin{array}{c}\mathrm{G} \\
(\%)\end{array}$ \\
\hline 1 & -1 & -1 & 13.6 & 67.4 \\
2 & -1 & 1 & 1.2 & 0.0 \\
3 & 1 & -1 & 60.2 & 97.4 \\
4 & 1 & 1 & 10.1 & 13.2 \\
5 & -1.41 & 0 & 0.2 & 3.2 \\
6 & 1.41 & 0 & 41.5 & 83.8 \\
7 & 0 & -1.41 & 58.3 & 93.5 \\
8 & 0 & 1.41 & 0.1 & 0.0 \\
9 & 0 & 0 & 13.1 & 71.9 \\
\hline
\end{tabular}

The criteria to interrupt the simulations was RMS residuals less than $10^{-5}$, oil mass imbalance of $10^{-4}$, and velocity was accompanied at the overflow to verify that the steady state was reached. In some simulations this happened at approximately 1.05 seconds (three times the residence time), so, to be conservative, the simulations were interrupted when all these criteria were accomplished and the total time of $1.13 \mathrm{~s}$ was achieved.

\section{RESULTS}

The responses of the factors analyzed on the experimental design are shown in Table 4.

Based on the results shown in Table 3 and on a statistical analysis following recommendations of Calado \& Montgomery (2003) and Montgomery (2012) with p-level of $5 \%$, equations 4 and 5 were found for the grade efficiency $G$ and the water flow ratio $R_{w}$. The squared correlation coefficient for $G$ and $R_{w}$ were 0.993 and 0.984 respectively.

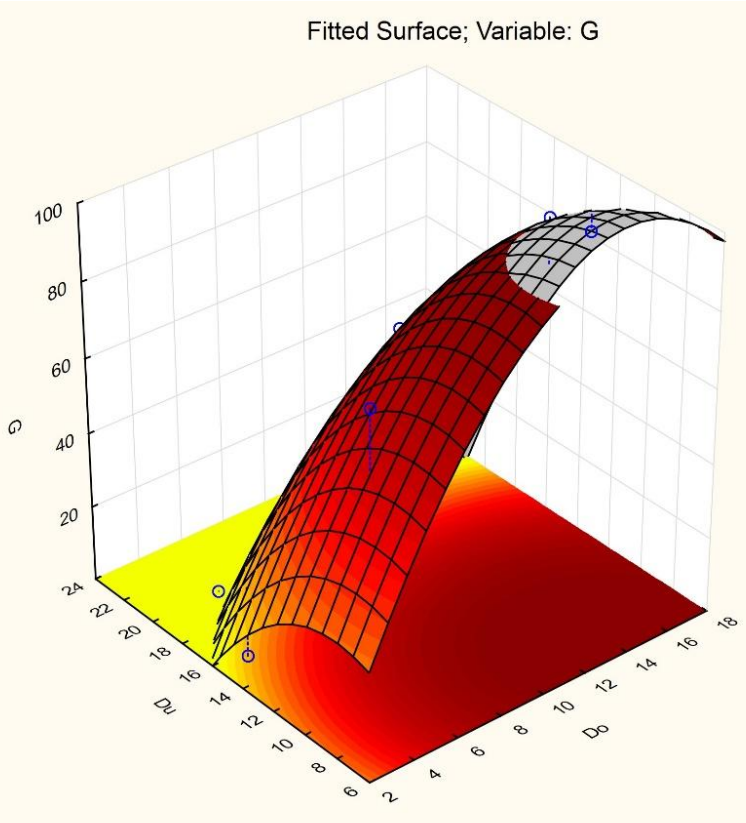

Figure 3. Response surface for the grade efficiency $\mathrm{G}$ (Do and Du are in $\mathrm{mm}$ ). 


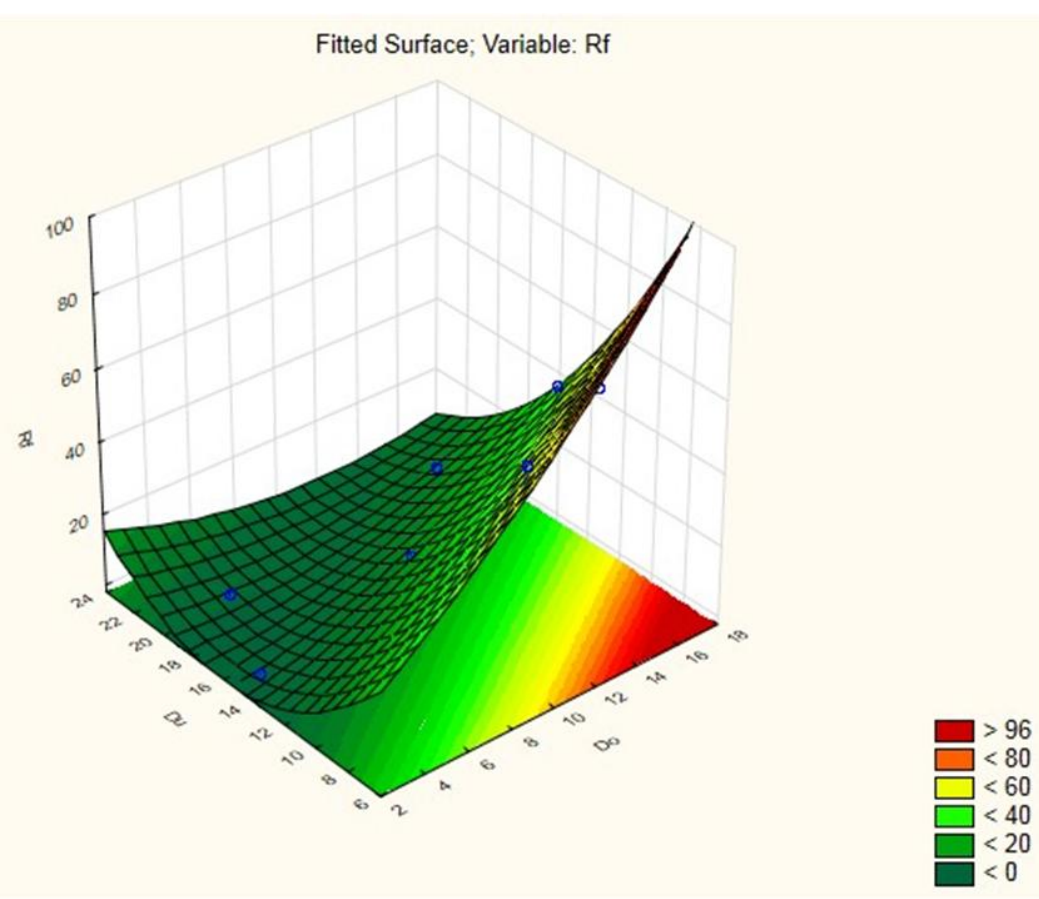

Figure 4. Response surface for the water flow ratio $R_{w}$ (Do and Du are in $\mathrm{mm}$ ).

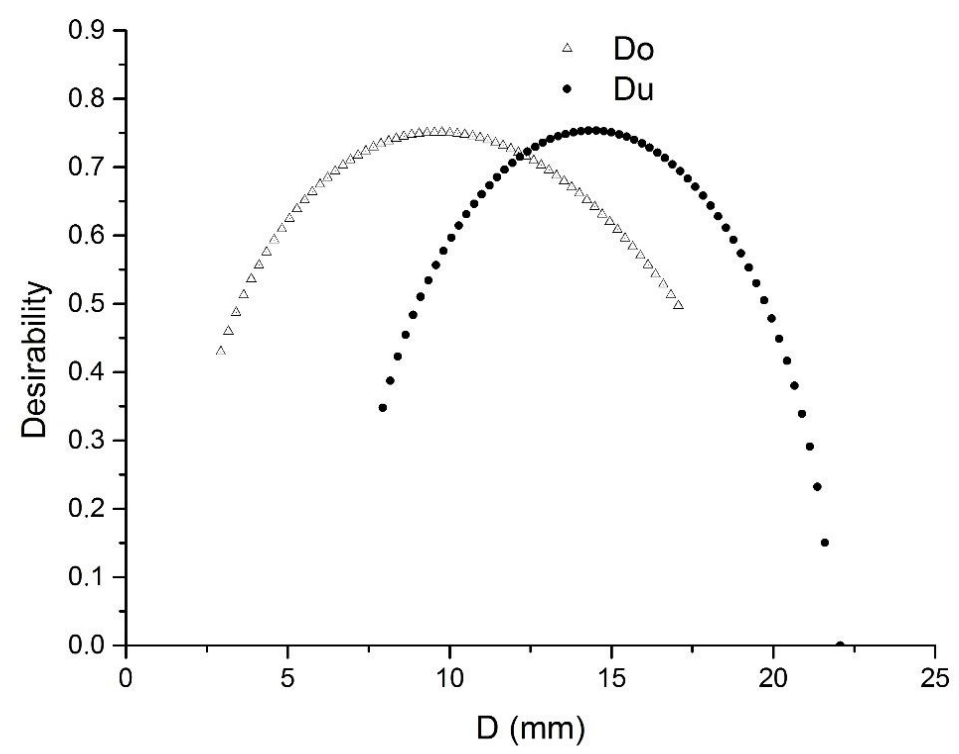

Figure 5. Desirability functions for $\mathrm{G}$ and $\mathrm{R}_{\mathrm{w}}$.

$G=71.91+18.38 D o-36.72 D u-$

$-13.73 D o^{2}-12.13 D u^{2}-1.70 D o D u$

$R_{w}=13.09+14.25 D o-18.11 D u+$

$+2.94 D o^{2}+7.11 D u^{2}-9.44 D o D u$
(4)

Where Du and Do are in millimeters.

Figures 3 and 4 show the 3D plot of the grade efficiency $G$ and the water flow ratio $R_{w}$, respectively, as a function of overflow and (5) underflow diameters. The diameter units are in millimeters. 

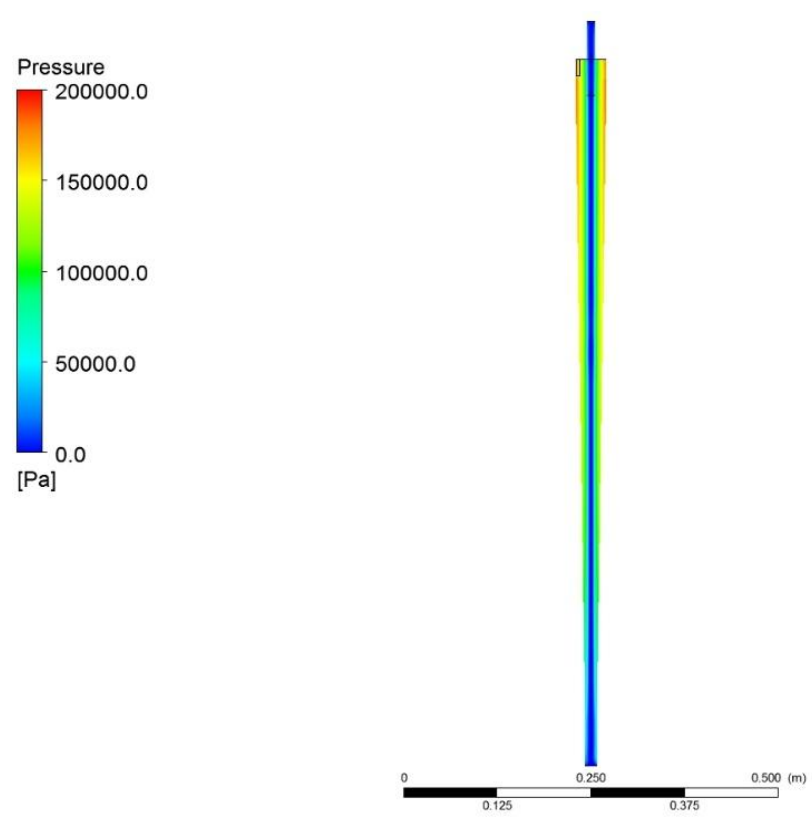

Figure 6. Manometric pressure profile for simulation 9.
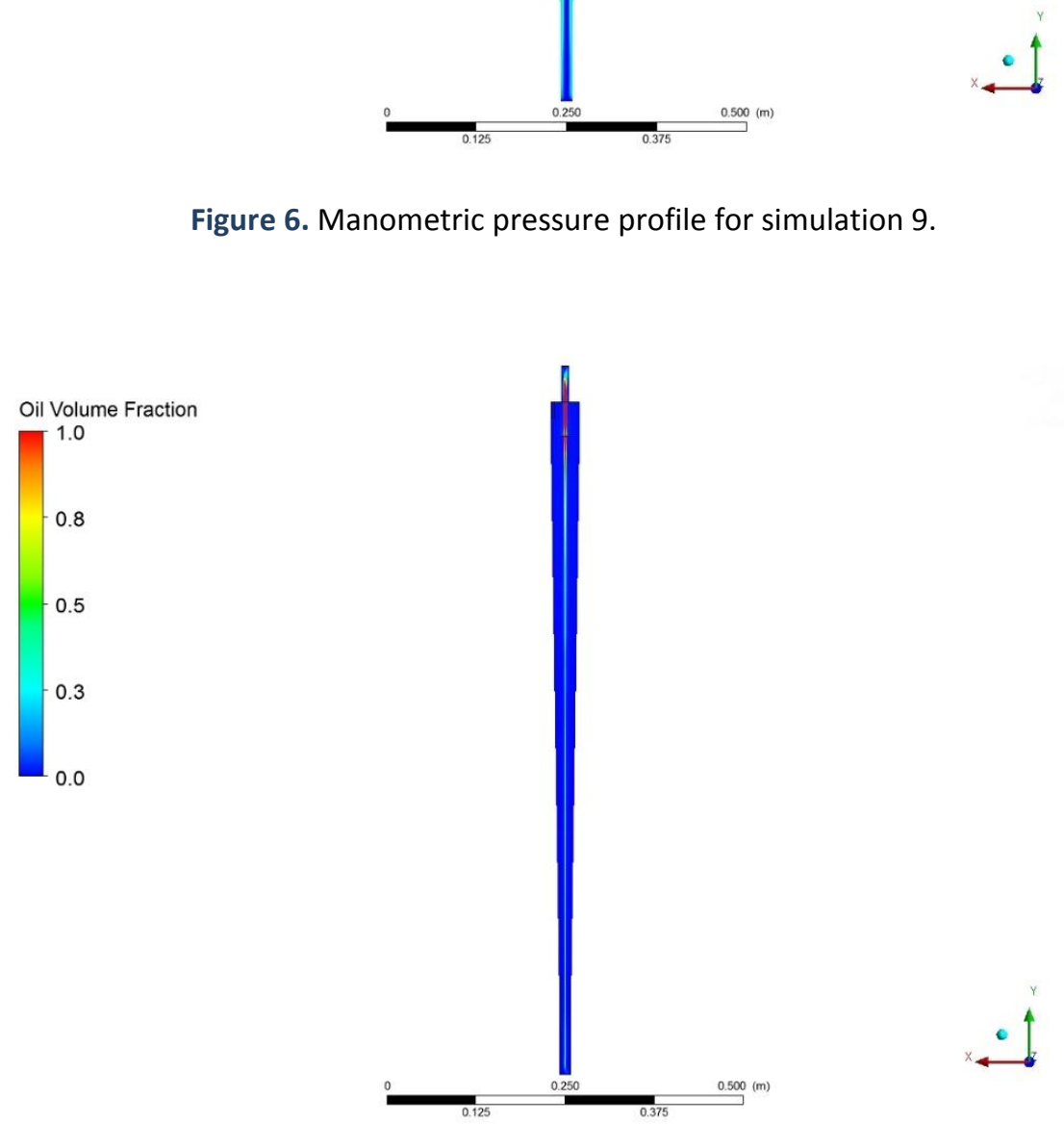

Figure 7. Volume fraction profile for simulation 9.

Figures 3 and 4 show no saddle points, but indicate that for a given pair of values of Do and $\mathrm{Du}$ results a local maximum for $G$, and that for another pair of values of Do and Du results a local minimum for $\mathrm{R}_{\mathrm{w}}$.

Desirability functions were used to find the values of Do and Du that maximize $G$ and minimize
$R_{w}$ simultaneously. To use these functions, first, it is necessary to specify a desirability score, where 0 is very undesirable and 1 is very desirable. The individual desirability scores for the predicted values for each dependent variable are then combined by computing their geometric mean (StatSoft, 2013). On this work, the desirability 
score for the variable $\mathrm{G}$ was set 0 for $0 \%$ and 1 for $100 \%$ and the desirability score for the variable $R_{w}$ was set 1 for $0 \%$ and 0 for $100 \%$.

Figure 5 shows the results of the desirability functions for grade efficiency and water flow ratio. The values of Do and Du that maximize $G$ and minimize $R_{w}$ are $10 \mathrm{~mm}$ and $15 \mathrm{~mm}$, respectively. These are the values of the central point of the experimental design and they produce a grade efficiency of $72 \%$ for a $25 \mu \mathrm{m}$ oil drop size and a $13 \%$ water flow ratio, which results in a reduced grade efficiency of $68 \%$ for this drop size.

Figures 6 and 7 show manometric pressure and volume fraction profiles for simulation 9. As expected by Thew and Nezhati, (1987), on the area near the wall the pressure is higher than at the central area of the equipment. For volume fraction, the less dense fluid concentrates at the center of the hydrocyclone and exits at the overflow, the more dense fluid is, otherwise it concentrates at the area near the wall and exit at the underflow.

\section{CONCLUSIONS}

In this work, simulations using a rotatable central composite design with response surface methodology for CFD simulations were carried out seeking to improve hydrocyclone geometry for oil/water separation.

The results show that, by increasing the value of overflow diameter, the values of grade efficiency and water flow ratio increase. Also, by increasing underflow diameter, grade efficiency and water flow ratio decrease.

For the hydrocyclone studied, the best values for overflow diameter and underflow diameter were 10 and $15 \mathrm{~mm}$, respectively, which produced a $13 \%$ water flow ratio, and a grade efficiency of $72 \%$ for a $25 \mu \mathrm{m}$ oil drop size.

\section{ACKNOWLEDGEMENTS}

The authors gratefully acknowledge the financial support provided by PETROBRAS, FINEP, CNPq, and CAPES.

\section{REFERENCES}

Bai, Z. S.; Wang, H. L.; Tu, S. T. Experimental study of flow patterns in deoiling hydrocyclone. Minerals Engineering, v. 22, p. 319-323, 2009. http://dx.doi.org/10.1016/i.mineng.2008.09.003

Bernardo, S., Mori, M., Peres, A., Dionisio, R. 3D computational fluid dynamics for gas and gasparticle flows in a cyclone with different inlet section angles. Powder Technology, v. 162 (3), p.190-200, 2006.

http://dx.doi.org/10.1016/i.powtec.2005.11.007

Calado, V., Montgomery, D. C., Experimental design using Statistica. E-Papers Editorial services, Rio de Janeiro, 260p., 2003. (in Portuguese)

Chuah, T., Gimbun, J., Choong, T.S. A CFD study of the effect of cone dimensions on sampling aerocyclones performance and hydrodynamics. Powder Technology, v. 162, p.126-132, 2006. http://dx.doi.org/10.1016/i.powtec.2005.12.010

Delgadillo, J.A.; Rajamani, R.K.; Exploration of hydrocyclone designs using computacional fluid dynamics. Int. J. Miner. Process., v.84, p.252-261, 2007. http://dx.doi.org/10.1016/i.minpro.2006.07.014

Dirgo, J,; Leith, D.; Cyclone collection efficiency: comparison of experimental results with theoretical predictions, Aerosol Science and Technology, v. 4, p.401-415, 1985. http://dx.doi.org/10.1080/02786828508959066

Elsayed, K., Lacor, C. The effect of cyclone inlet dimensions on the flow pattern and performance. Applied Mathematical Modeling, v.35, p.19521968, 2011.

http://dx.doi.org/10.1016/j.apm.2010.11.007

Freitas, A.G.B., "Modeling and simulation of oily water treatment using hydrocyclones". M.Sc. dissertation, Chemical Engineering Program, Federal University of Sergipe, São Cristóvão, 124p., 2009. (in Portuguese)

Freitas, A.G.B.; Paixão, A.E.A.; MEdronho, R.A.; Silva, G.F.; Influence of some hydrocyclone geometric variables on the flow ratio and the reduced grade efficiency: a CFD Study. In: ENEMP 2009 - XXXIV Brazilian Conference on Particulate Systems, Proceedings of the ENEMP 2009. UNICAMP, Campinas, 2009. (in Portuguese) 
Gibson, M. M., Launder, B. E. Ground effects on pressure fluctuations in the atmospheric boundary layer. Journal of Fluid Mechanics, v. 86, part 3, p . 491-511, 1978.

Griffiths, W.D., Boysan, F. Computational fluid dynamics (CFD) and empirical modeling of the performance of a number of cyclone samplers, Journal of Aerosol Science, v. 27(2), p.281-304, 1996. http://dx.doi.org/10.1016/0021-8502(95)00549-8

Gupta, A., Kumar, R. Three-dimensional turbulent swirling flow in a cylinder: Experiments and computations. International Journal of Heat and Fluid Flow, v. 28 (2), p. 249-261, 2007. http://dx.doi.org/10.1016/j.ijheatfluidflow.2006.04.005

Huang, L., Kumar, K., Mujumdar, A.S. Simulation of a spray dryer fitted with a rotary disk atomizer using a three-dimensional computational fluid dynamic model. Drying Technology, v.22 (6), p.1489-1515, 2004. http://dx.doi.org/10.1081/DRT$\underline{120038737}$

Kaya, F., Karagoz, I. Performance analysis of numerical schemes in highly swirling turbulent flows in cyclones. Current Science, v.94 (10), p. 1273-1278, 2008.
Montgomery, D. C. Design and Analysis of Experiments. $8^{\text {th }}$ Edition. Wiley, 752p., 2012.

Sommerfeld, M., Ho, C.A. Numerical calculation of particle transport in turbulent wall bounded flows. Powder Technology, v.131 (1), p.1-6, 2003. http://dx.doi.org/10.1016/S0032-5910(02)00293-0

StatSoft, Inc.. Electronic Statistics Textbook. Tulsa, OK: StatSoft. (2013) Available at: <http://www.statsoft.com/textbook/>.

Svarovsky, L. Solid-liquid Separation. Czech Repuplic: Butterworth-Heinermann, p. 191-245, 2000.

Thew, M. T., K. Nezhati. Aspects of the performance and scaling of hydrocyclones for use with light dispersions. 3rd International Conference on Hydrocyclones. Oxford: Elsevier Applied Science Publishers, p.167-180, 1987.

Wan, G., Sun, G., Xue, X., Shi, M. Solids concentration simulation of different size particles in a cyclone separator. Powder Technology, v.183, p.94-104, 2008.

http://dx.doi.org/10.1016/j.powtec.2007.11.019 